\title{
FIT AND PRESSURE COMFORT EVALUATION ON A VIRTUAL PROTOTYPE OF A TIGHT-FIT CYCLING SHIRT
}

\author{
Yetanawork Teyeme ${ }^{\star 1,2}$, Benny Malengier ${ }^{1}$, Tamrat Tesfaye $^{2}$, Simona Vasile $^{3}$ and Lieva Van Langenhove ${ }^{1}$ \\ 1 Ghent University, Faculty of Engineering and Architecture, Department of Materials, Textiles and Chemical Engineering, Technologiepark 907, \\ 9052 Zwijnaarde, Belgium \\ 2 Bahir Dar University, Ethiopian Institute of Textile and Fashion Technology, Bahir Dar, Ethiopia \\ 3 Fashion and Textiles Innovation Lab (FTILab+), HOGENT University of Applied Sciences and Arts, Belgium \\ Corresponding Author. E-mail: YetanaworkWubneh.Teyeme@UGent.be
}

\begin{abstract}
:
Graduated compression is widely used for medical application to prevent perioperative venous thromboembolism, but other applications such as sportswear can potentially also benefit from it. A tight-fit cycling shirt meant to ensure the correct position during cycling and prevent injuries was designed. The aim of this study was to improve garment pattern design from the aspect of clothing pressure for providing support and enhancing comfort to the user. This paper investigates the suitability of pressure maps from 3D fashion design software CLO $3 D$ for design and in particular its capability to discriminate between various materials and cycling postures. Moreover, the impact of the mechanical properties of fabric was analyzed. In particular, virtual prototyping tool CLO 3D and pressure mapping were employed to achieve the required graduated compression while ensuring fit and comfort. Pattern adjustments were iteratively performed until stress, strain, and pressure maps showed adequate fit and pressure of the cycling garment on the virtual cyclist in static and dynamic cycling positions. The impact of fabric types on garment fit has been shown by generating the stress, strain, and pressure maps with a virtual simulation. It was found that the visualized pressure on the human body model shows distributions that are related to contact between body and garment, and large compression stresses occur in the lower parts of the two shirts. Evident garment deformation was shown at hip level, upper arm, lower front side seam, and front neck, which can reduce garment wear comfort and freedom of movement. The output was found to be sufficiently accurate to optimize the garments based on material and cycling posture.
\end{abstract}

\section{Keywords:}

stretch fabric, pressure, virtual prototyping, CLO 3D, pattern modification, tight-fit sportswear, cycling posture

\section{Introduction}

Garment fit and pressure comfort have been identified as among the important attributes for tight-fit sportswear [1]. Compression is a result of forces generated during dynamic interactions between the body and forces involved in the garment strain and consequently induce pressure sensations. Therefore, both the applied pressure and the way pressure changes during use are important to understand. The degree of compression exerted by a garment is dependent on the individual size and shape of the part of the body to which it is applied, as well as the construction and fit of the garment and mechanical properties of the fabrics. Garment pressure comfort has been identified as one of the important attributes. It has a great impact on overall clothing comfort, and if the pressure is too high or too low, it does not meet functionality requirements. Tight-fit sportswear is reported to provide the body with suitable support and compression to accelerate blood circulation, hence improving sports performance, improving posture, and preventing injury [1-5]. Since the 19th century, compression therapy has been widely used [6-8]. The use of graduated compression has been proven beneficial, specifically as it works with the muscles to encourage blood flow toward the heart [9]. Li et al. (2005) analyzed the possible reasons influencing the stocking pressure performances and found skin pressure distributions and magnitudes applied by graduated compression stocking were significantly determined by the specific locations of testing points and potentially influenced by the pressure sensor and testing methods [10]. Clothing pressure can be assessed by many different kinds of methods, including theoretical calculations [11], simulation [12, $13]$, and direct and indirect tests [14, 15].

Garment pressure is normally measured by instruments initially used in compression bandages including PicoPress (MICROLAB ELETTRONICA S.a.s. di Bergamo Giorgio \& C., Italy) [16] and Kikuhime (TT Medi Trade, Soro, Denmark) [17]. However, they are able to measure the pressure of the garment only on the skin locally, at a limited number at locations. Visualization of the pressure distribution on a virtual manikin would offer many advantages including higher accuracy of garment fit prediction without really trying it on and visualized pressure distribution on the human body, both of which are related to contact between the garment and the body, information that can be useful for the mechanical functional 
design of apparel. Besides, it is advantageous to evaluate the overall fit level for all relevant positions and to determine static and dynamic performance of the selected fabric, as well as garment fit effects in terms of comfort and fabric properties [18, 19].

Clothing modeling and virtual trying on have grown from basic shape modeling (2D) to 3D apparel. Therefore, 2D apparel CAD technology is being extended to $3 D$. At present, 3D CAD software programs are available responding to the demand of virtual prototyping, and $2 \mathrm{D} \mathrm{CAD}$ is made to measure (MTM) the pattern design system in the clothing industry. This new technology includes $\mathrm{V}$ stitcher ${ }^{\mathrm{TM}}$ by Browzwear (Browzwear ${ }^{\circledR}$ Solutions Pte. Ltd, Singapore; 3D Runway from OptiTex, Ltd, Israel; AccuMark 3D from Gerber Technology, Tolland, USA; TUKA3D (Tukatech Inc., USA); Modaris 3D Fit from Lectra Paris, France; and Vidya from Assyst, Germany. More recently, two other solutions were added with similar capabilities, namely, CLO 3D (CLO Virtual Fashion LLC, Korea), and Marvelous Designer (CLO Virtual Fashion Inc.) [20-22]. These new technologies offer greater efficiency and time-saving solutions for many complex tasks with higher accuracy and lower cost. Such a system commonly comprises three modules: (1) a $3 \mathrm{D}$ parametric mannequin module used to create various personalized 3D human models rapidly from real body measurements according to specific customers' body shapes and dimensions; (2) a fabric property module used to adjust the virtual fabric according to real fabric properties, which enables the evaluation of the interaction of a particular type of fabric with the 3D body form; and (3) a virtual pattern sewing module to sew garment patterns together on specific 3D human models, taking into account the performance of the virtual fabric [23, 24]. Moreover, the pressure maps obtained from the systems could also potentially permit the assessment of pressures without creating physical samples. The development of such tools is hence advantageous in the compression sportswear market, though companies are not now claiming accuracy in this area.

Pressure applied on clothing varies according to the design, pose of the wearer, garment material, the wearer's body shape, and so on. Changes of anthropometric body measurements [25] with posture have been demonstrated [26], changes of the clothing pressure with posture have also been reported, and body postural changes have significantly influenced the skin pressure profile applied by compression stockings tested in different body positions [27].

The ultimate purpose of the present research is to develop a customized tight-fitting cycling shirt prototype, with a graduated compression ensuring various body pressure distribution and an embedded sensor to monitor and limit the range of motions causing potential back pain. This paper investigates the suitability of pressure maps from CLO 3D for design and in particular its capability to discriminate between various materials and cycling postures.

\section{Clothing ergonomics}

Clothing ergonomics gives full consideration to the human body and the ability of clothing to comfort. The focus is the study of the relationship between humans and their clothing, focusing on anthropology, genetics, anatomy, body shape, physiology, biomechanics, and psychology.

\subsection{D Human body model}

A 3D model, 0.43 meters in width (across shoulder length), 1.89 meters in height, and ID male_B _V3.avt, in standard A-pose avatar (Figure 1) was selected from the software library.

\subsection{Virtual prototyping}

It is believed that $3 \mathrm{D}$ simulation of pressure garments during the product development process would be a particularly useful tool to quickly assess garment fit and change it when necessary, eliminating the need for several physical prototypes and related pressure testing.

The 3D simulation was created using the CLO 3D software and fabrics from the library. The garments' pattern construction from 3D simulation was executed in two stretch fabrics in order to measure the pressure model. If the simulation model is successful, this can next be used as a tool in the product development process of compression garments. It will facilitate

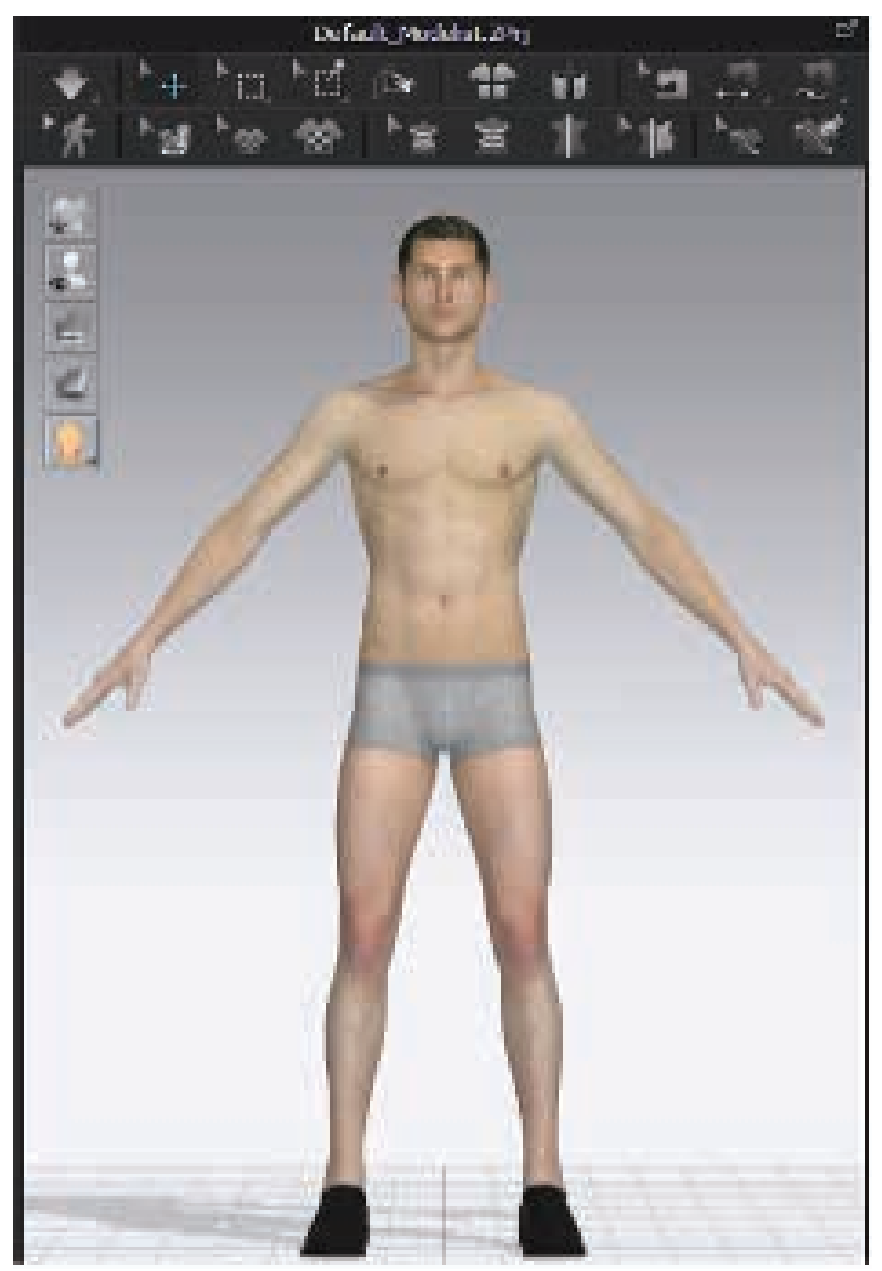

Figure 1. Model Avatar 
understanding of the effect of fabric, garment measurements, and construction on compression without expense and with less time creating the garments.

\subsection{Mechanical properties of knitted fabrics}

The selection of fabrics available in the library aimed at diversity of samples, including fiber content, structure, thickness, buckling, shear, elongation, and weight. Elastic fabric is selected, which is of great importance in order to produce tight-fitting clothing with comfortable properties and good body fit [28]. Not only size and shape of the part of the body influence the value of clothing pressure comfort but also the mechanical properties of stretch fabrics that change the value of the pressure when it is worn [29]. The mechanical properties of several stretch knit fabrics that will be used for the physical garment prototype were tested. However, the real values of the mechanical properties of fabric are determined by common testing instruments that cannot be uploaded to the library of CLO 3D simulation software directly. The software provides relative values of the mechanical properties of fabric automatically for the fabric chosen. Therefore, two fabrics A and $B$ were selected from the library to be used in the simulation of the virtual prototype. Their mechanical properties are listed in Table 1. The material composition of fabric $\mathrm{A}$ is $68 \%$ viscose, $27 \%$ nylon, and $5 \%$ elastane with a thickness of $0.30 \mathrm{~mm}$ and $330.89 \mathrm{~g} / \mathrm{m}^{2}$ mass per unit surface, and fabric $\mathrm{B}$ is $100 \%$ cotton with a thickness of $0.64 \mathrm{~mm}$ and $163.94 \mathrm{~g} / \mathrm{m}^{2}$ mass per unit surface. Although cotton is not suitable for the scope of compression sportswear, it was selected because it is easier to assess the differences and capabilities of the software in terms of pressure maps. Fabrics with a higher percentage of elastane would be desirable, but they are not available in the library.

\subsection{Workflow}

The virtual prototyping process covers several work steps, starting with a 2D pattern preparation and ending with the development of a final virtual prototype (Figure 2). The first step is the export of a 2D garment patterns file (which was developed using LECTRA MODARIS, a 2D graphics software) into CLO $3 \mathrm{D}$. MODARIS enables the development of $2 \mathrm{D}$ pattern pieces. After the development of a $2 \mathrm{D}$ pattern, CLO 3D, which is like a Modaris 3D Fit, we need to test the fitting of the garment by virtually sewing the pieces together and trying different fabrics and textile designs. In this way, pattern pieces are shown on the artboard (Figure 3), and the technical characteristics of the flat patterns can be manipulated directly. The garment represents a men's cycling outfit, size medium with full front zipper, three rear pockets, an elastic waist, kimono short sleeves, and generous side panels. Second, an A pose avatar 3D model was chosen (Figure 1).

The third stage was the virtual sewing of the prototype. It enables to visualize flat pattern placed around a 3D virtual body (men avatar) using a "body map". As a result, they become attached with seams on the borders as specified, attaining the shape influenced by the shape of the body. The garment was constructed over a virtual human body avatar according to the human body feature lines and the desired garment style. Figure 3 describes the process of flat patterns visualization and prototype assembly through seam simulation.

In the fourth step, material properties are assigned to different pattern pieces and regions. The physical parameters configuration of the fabric in a virtual environment are established to achieve better simulation. For this, the real fabric sample should be used. However, since we could use only predefined fabrics for virtual simulation in CLO 3D, this step is seen as a drawback of this study, especially due to the limited number of high stretch fabric available. However, the purpose here is to evaluate the software capability to show different

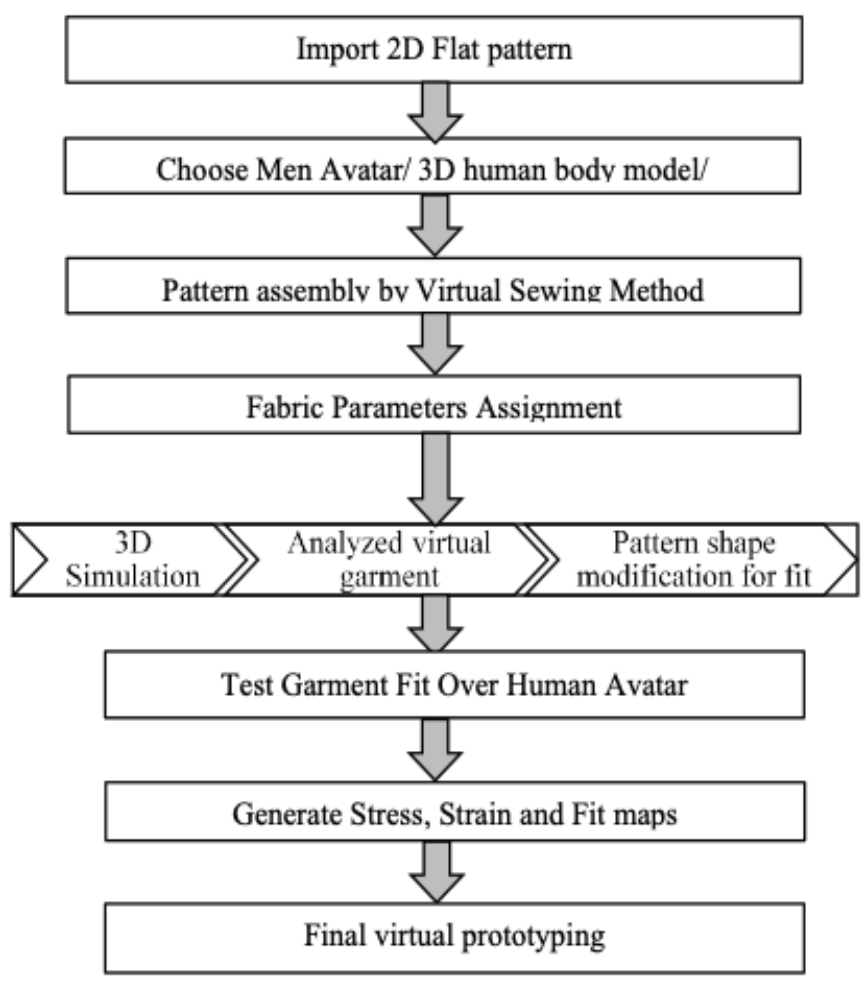

Figure 2. Workflow of virtual prototype preparation

Table 1. Values of fabric mechanical properties for the measurement of clothing pressures

\begin{tabular}{|c|c|c|c|c|c|c|c|c|c|c|c|c|}
\hline Fabric & BSF & BSP & BRF & BRP & BF & BP & SF & SP & SH & DE & ID & FC \\
\hline A & 80 & 80 & 90 & 90 & 30 & 30 & 25 & 25 & 15 & 10 & 1 & 0 \\
\hline B & 80 & 80 & 85 & 85 & 43 & 48 & 26 & 28 & 21 & 13 & 1 & 3 \\
\hline
\end{tabular}

Note: BSF is buckling stiffness-weft; BSP is buckling stiffness-warp; BRF is buckling ratio-weft; BRP is buckling ratio-warp; BF is bending-weft; BP is bending-warp; SF is stretch- weft; SP is stretch-warp; SH is shear; DE is density; ID is internal damping; FC is friction coefficient. The fabric mechanical properties are relative values in the range (1-99), defined by the software. 


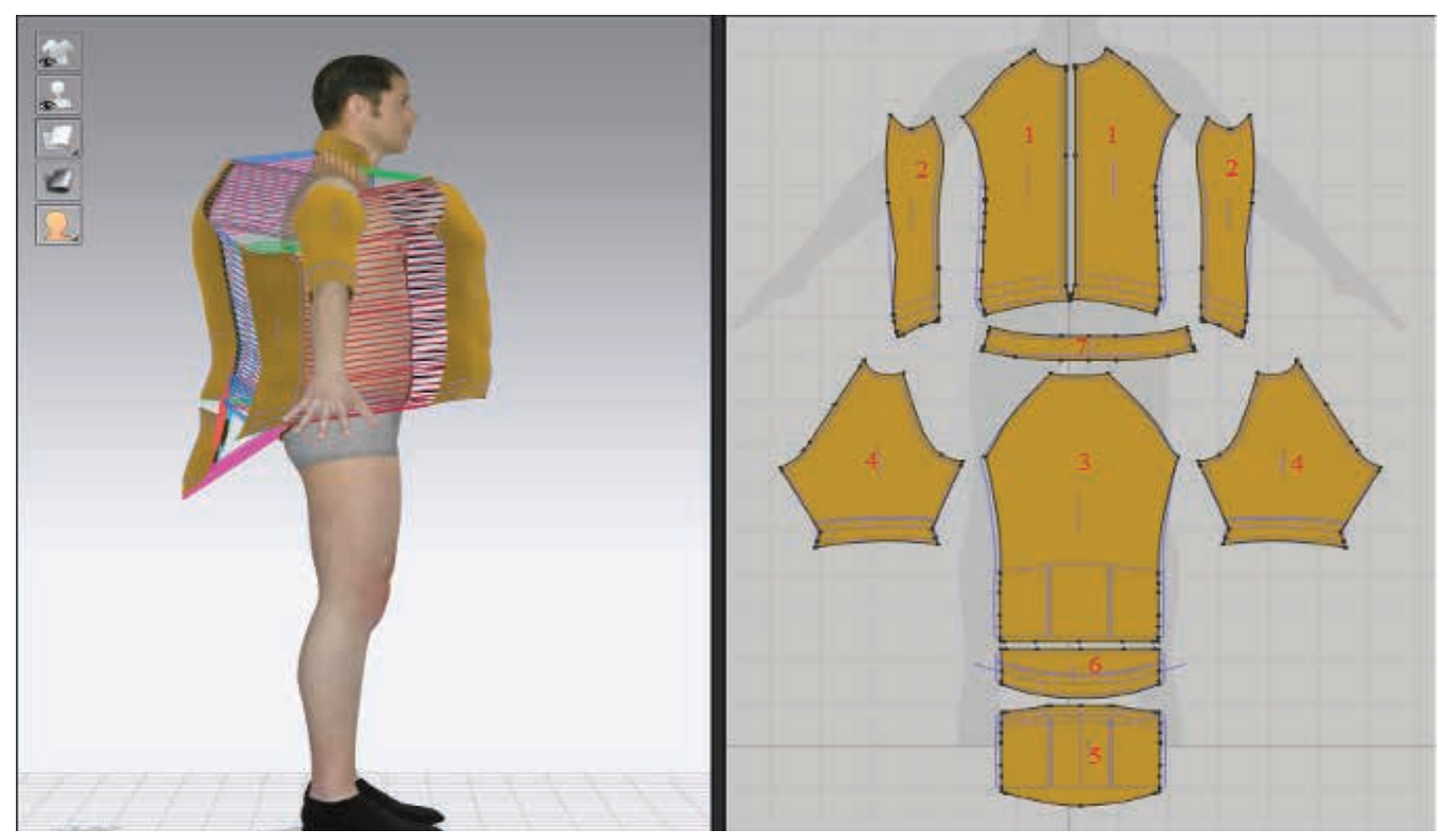

Figure 3. Original flat pattern pieces visualization (picture right) and prototype assembly process on the artboard (picture left)

pressure maps due to different material. The properties of the fabrics $A$ and $B$ selected are shown in Table 1.

Simulation and analysis of virtual garment fit and pressure at different body locations: Once the virtual garment has been assembled and placed roughly on the avatar customized based on standard human body measurements, the real process is simulated in the avatar window using the CLO $3 D$ interface.

Analysis of garment fitting has been done through stress, strain, and fit maps, created during the simulation process for the garment prototype over the human model avatars. For digital clothing pressure and evaluation, three image typologies were extracted and analyzed, namely: the stress map; strain map and fit/realistic map, both in a static A-pose (Figure 1) and in a dynamic cycling position (Figure 8). Through color diagrams, the simulation map indicates aspects related to the ergonomics of the parts with the areas of greater and lesser tension in the simulated fabric [30, 31].

The Stress Map (Figure 4a) shows the external stress causing garment distortion by area of the fabric and appears in an arrangement of colors with the number where the red color indicates the highest stress while the blue color indicates zero distortion, $100 \mathrm{kPa}$ and $0.00 \mathrm{kPa}$, respectively. The unit of digital clothing pressures is Virtual $\mathrm{kPa}$, which is not similar to the unit of real pressure $\mathrm{kPa}$, according to the CLO 3D manual.

The Strain Map (Figure 4b) represents the rate of clothing distortion due to external stress. This stress is quantified as a percentage and is represented by a color diagram with eight colors: red indicates $120 \%$ of the distortion rate while blue indicates $100 \%$ (no distortion).

The Fit Map (Figure 4c) shows how tight clothes are on the user's body. This is shown through a color scheme overlaid on the simulation, where red indicates the impossibility of wearing the garment, orange indicates a very tight area, yellow a slightly tight area, and a transparent part indicates that the area is loose.

\section{Results and discussion}

We evaluated a series of local fit levels and pressure at certain critical contact areas of the body with a tight-fitting cycling shirt. The evaluation shows impact on the body area with respect to the mechanical properties of the fabric and pattern shape, whichare changed and influence garment fit. Hence, the measurements obtained of this pressure are critical indexes to guide garment design. Therefore, we mainly focused on a discussion of the pressure range on the body caused by the garment. The three map types generated during the simulation process for the fabric over the parametric avatar show differences in garment fit and the areas of greater and lesser tension in the simulated fabric.

In order to analyze the change of clothing pressure for different human body regions, we divided the shirt patterns into 13 regions: the mid-hip/pelvis region, waist region, front neck region, side neck region, back neck region, upper-front chest region, lower chest region, upper back region, lower back region, front shoulder region, back shoulder region, upper lumbar region, lower lumbar region, and upper arm region.

The authors identified and compared the critical strain areas of the 3D surface as the mid-hip level, the upper chest, the shoulder, front neck region, the upper arm, and armpit. As shown in Figure 4, the simulation shows the influence of fabric type on garment fit and pressure maps. Evident garment deformation was present at the hip level, upper arm, lower front side seam, and front neck, and is shown in red, indicating that wearing it is impossible, which means a lack of garment wear comfort and freedom of movement. More body parts on the 


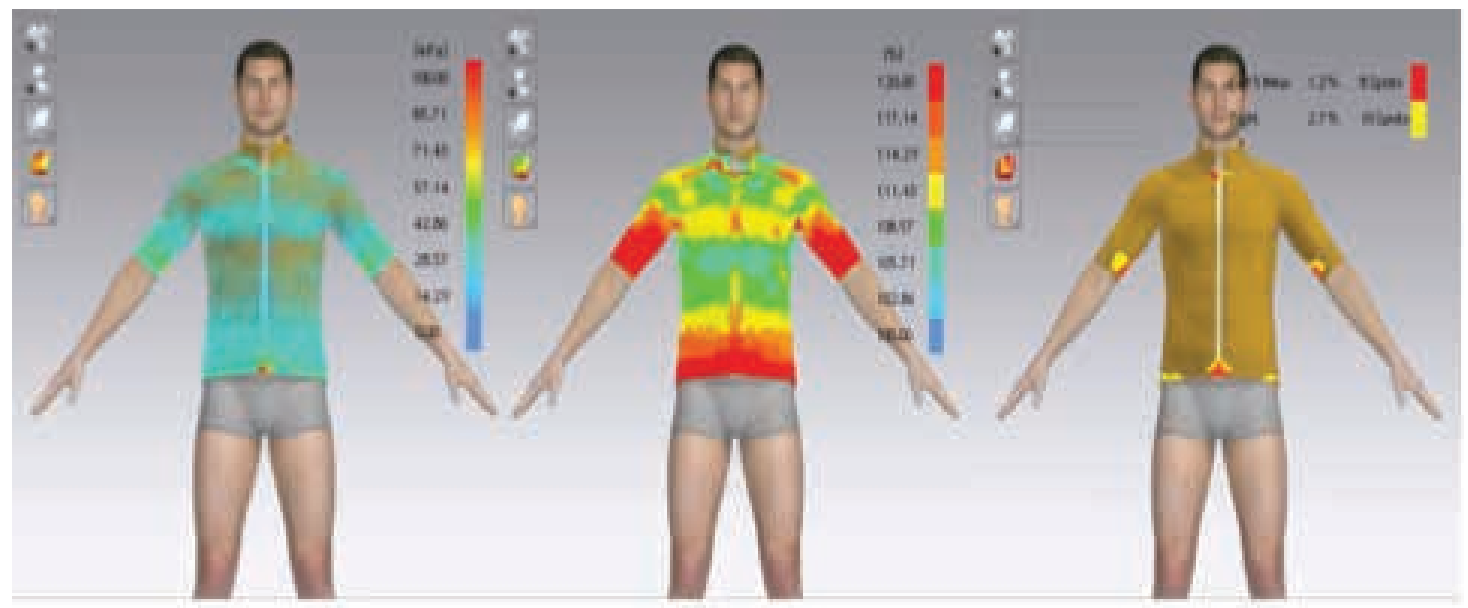

(a)

(b)

(c)

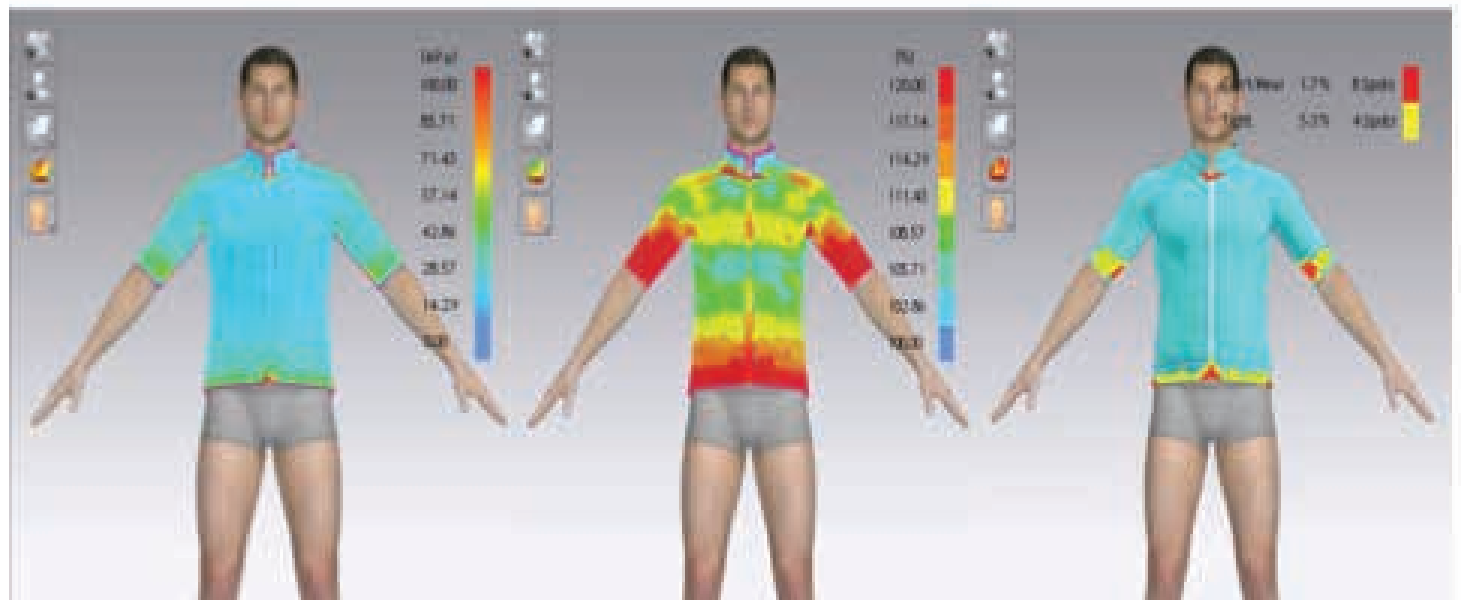

Figure 4. Stress (a), strain (b) and fit maps (c) of selected fabrics A (picture above) and B (picture bellow) respectively in static condition

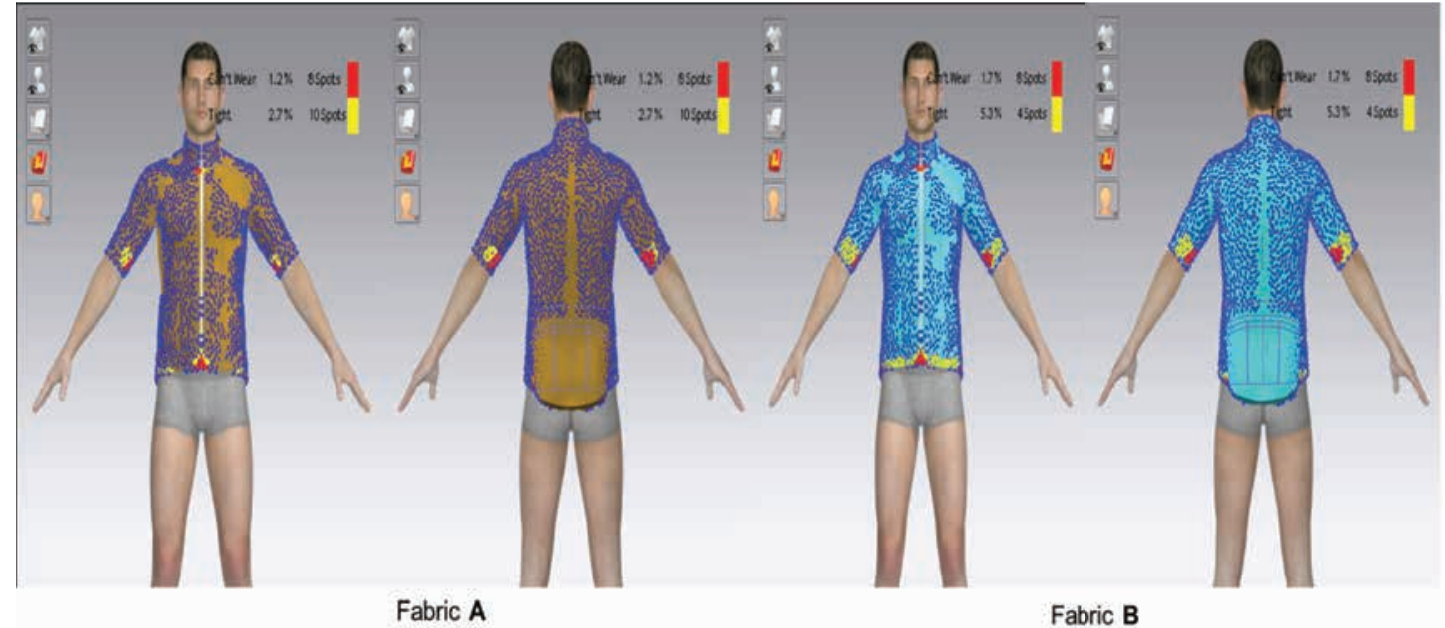

Figure 5. Clothing pressure in static condition with original pattern depending on fabric $A$ and $B$

same evaluation of garment fit are presented as a very tight area.

Furthermore, there are slightly tight areas (yellow) in eight spots including front side hip level, lower centre front, front left side neckline and lower seam line of upper arms. In the fit map evaluation (Figure 4c), the red indicates areas that are very tight and which restrict freedom of movement and comfort.
The static clothing pressures were determined with CLO 3D in the collar panel, front panels, sleeve panels, and side panels according to the result of the fit map in the standing posture (Figure 5). The collar, neckline, lower front region/hip level, and sleeve fit badly and result in high clothing pressures, so the patterns need great adjustments (i.e., eight spots of poor fit and four spots of tight fit area, represented with red and yellow, respectively, for both fabrics). As shown in Table 2, pressure points correspond to the lower and neckline front panels, and 
Table 2. Measured value of pressures based on different fabric (Unit: Virtual $\mathrm{kPa}$ )

\begin{tabular}{|c|c|c|c|c|}
\hline Fabric type & Sleeve & Front & Side panel & Collar \\
\hline A & 46.6 & 53.5 & 36.45 & 22.36 \\
\hline B & 58.5 & 70.5 & 49.4 & 28.07 \\
\hline
\end{tabular}

the lower side seam line and lower upper arm are excessively tight; pressures increase in the static condition.

Previous studies have demonstrated the influence of fabric mechanical properties on clothing pressure [32, 33]. Also, in this study, we measured pressure exerted by fabrics $A$ and $B$, with the original patterns, and the results show lower pressure in case of Fabric A, which can be due to entirely different mechanical properties of the two fabrics (Table 1), where $A$ is a stretch fabric.

As dynamic wear comfort is important for cyclists from an ergonomic perspective, adjustments were made to the patterns based on the dynamic clothing pressure. The adjustments consisted of modifying pattern pieces and adding ease of wear at specific points. The simulation, pressure evaluation, and adjustment process were repeated several times until the desired effect was reached, resulting in the final virtual prototype. Hence, ease of fit was incrementally added at the side seam line, front neckline, collar, center front seam line, and the lower sleeve seam line for reducing these parts' clothing pressure, until the result was satisfying (i.e., no tight fit or bad fit, which means no spot shown as red or yellow in the fit map). We also increased the length and width of the sleeve armhole, lower side panels, collar, and hip level of front panels in order to avoid wrinkles and stress in the seam line, as that leads to extra tension in the garment. The fit map after the optimization of the pattern is shown in Figure 6.

The clothing pressures were measured applying the customized pattern pieces according to mechanical properties of the fabric in the static standing posture (Figure 7).

From the discussion above, the garment has good comfort of wear and fit in static condition but will potentially lack comfort in the dynamic condition (Figure 8). This is because designers usually design clothing patterns by considering the static condition (i.e., body size charts are made based on anthropometric measurements taken in static posture) (Figure 1).

For future research, the authors are interested in making a prototype that can improve anatomical alignment and reduce the risk of cyclist back injuries through specially designed pressure areas under unwanted movement. In order to optimize the design of clothing in terms of such functional performance and pressure comfort, we ideally need to examine garment pressure distribution virtually, during the design phase. Hence, the amount of pressure on the panels and its effect on the compressibility of the garment in different body regions for both fabrics $A$ and $B$ were compared in static standing position and dynamic cycling posture with the original and modified patterns. The dynamic cycling posture was constructed for a road cyclist based on the ideal position on a bicycle (Figure 8 \& 9) [34-36].

The influence of patterns and posture on pressure is shown in Figures 10 and 11, respectively. To create these figures, the pressure distributions on static and dynamic positions at various body locations were measured five times, and the average values were taken for accuracy. As was already

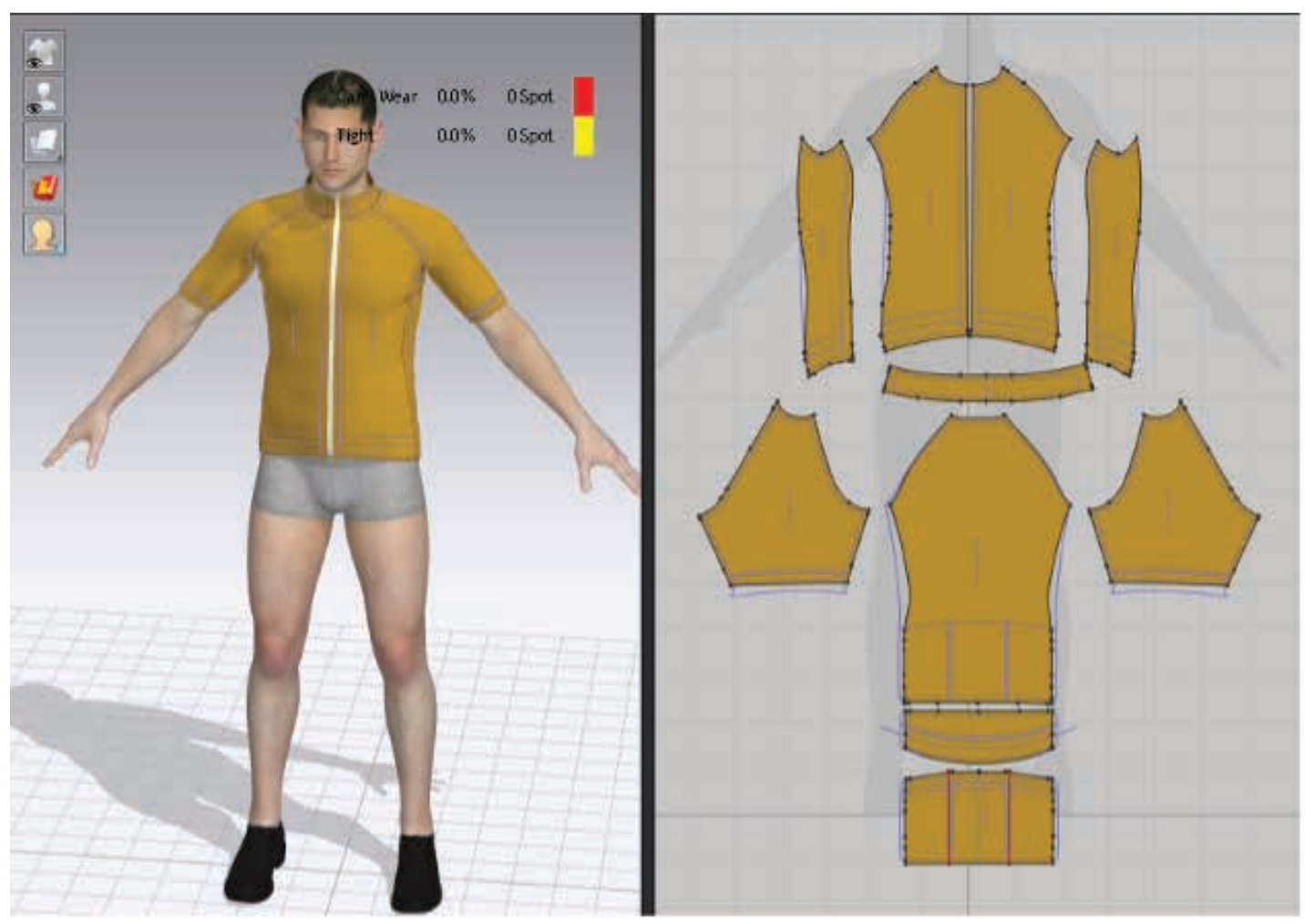

Figure 6. Fit map with optimized 2D pattern for fabric A or B 


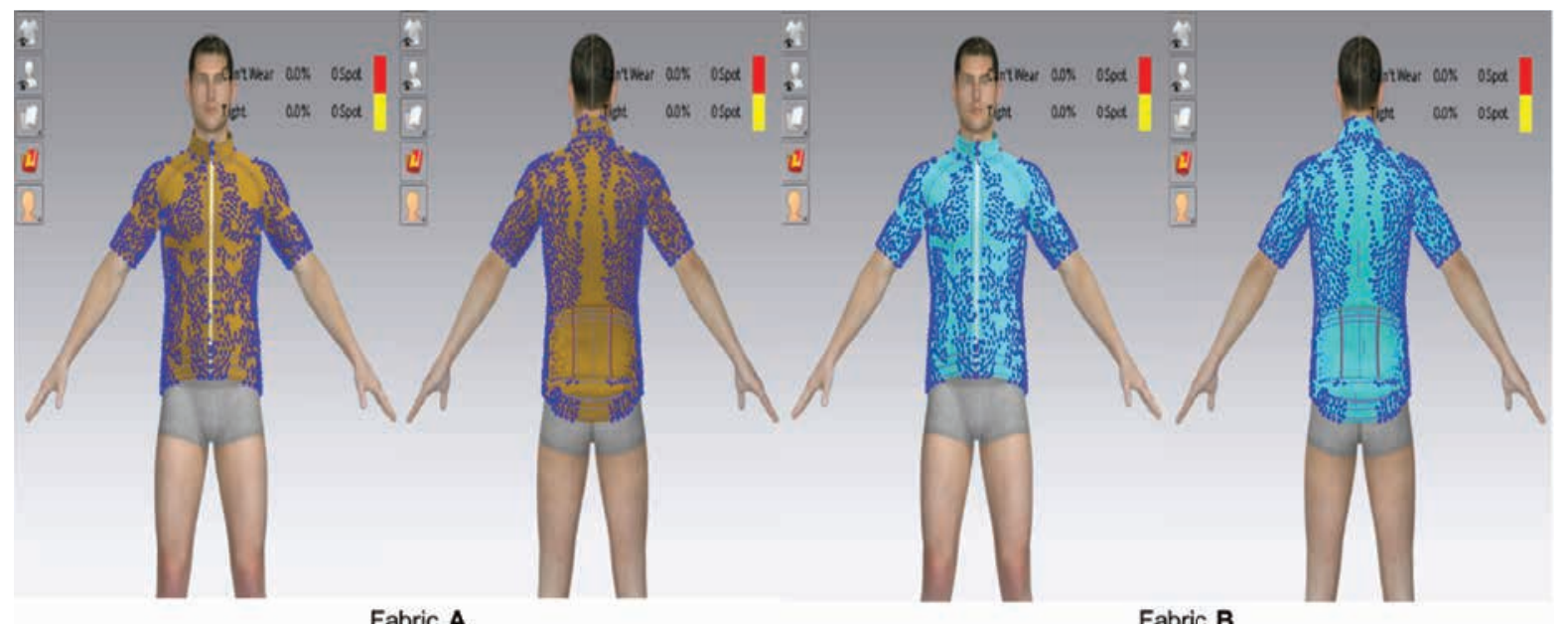

Figure 7. Simulated clothing pressure in static condition with optimized pattern

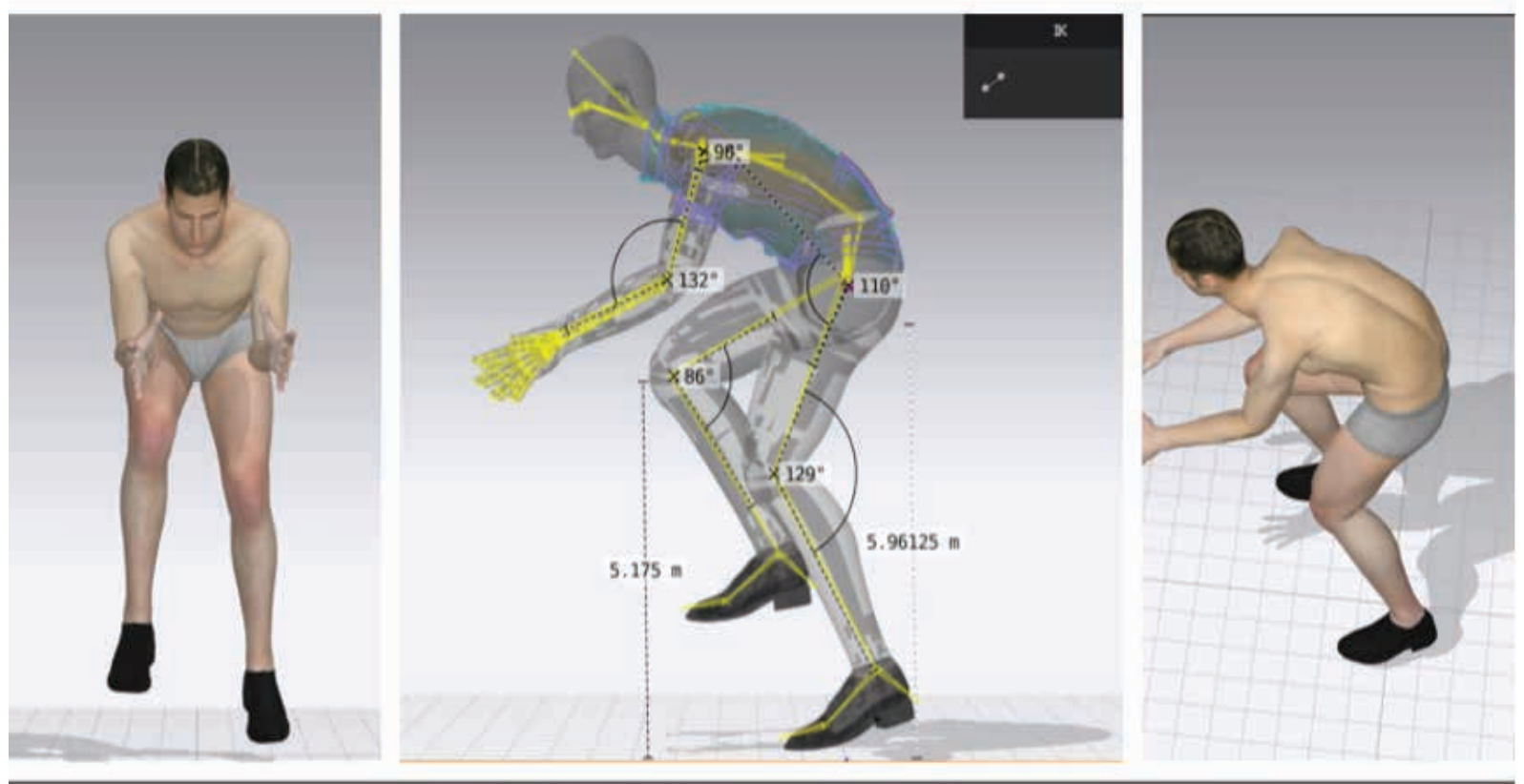

Figure 8. Correct dynamic cycling posture [32-34].

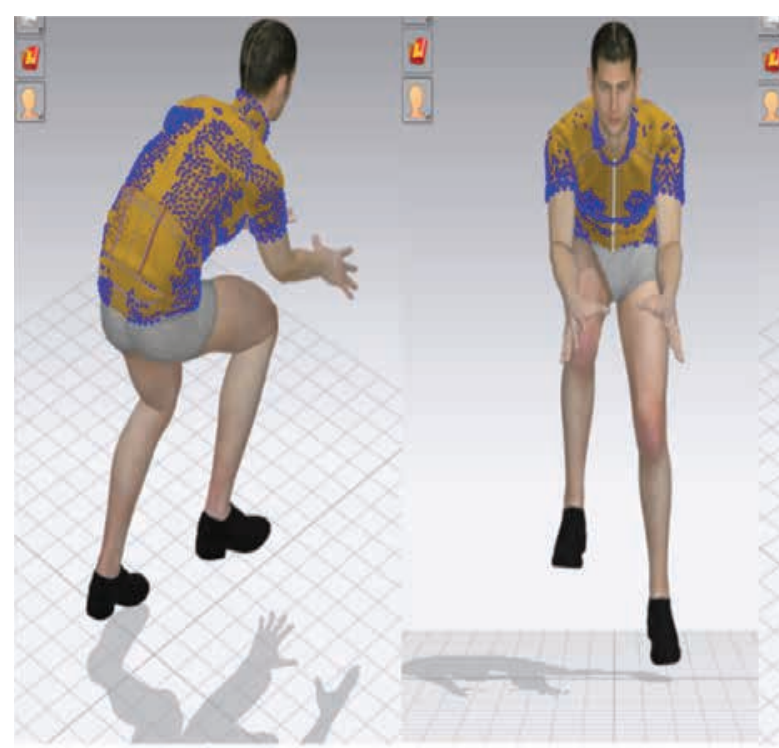

Fabric A

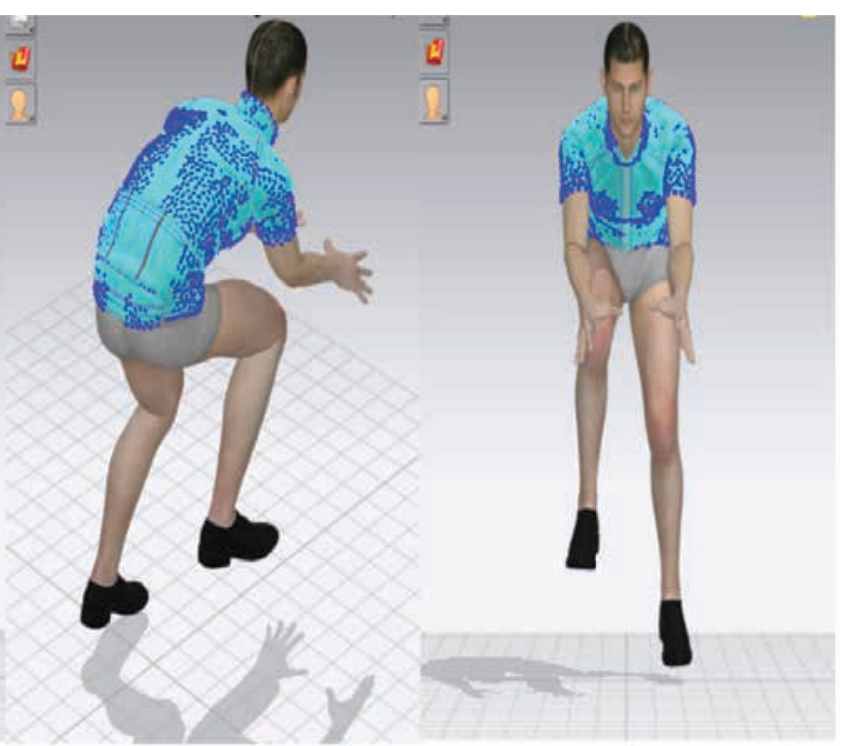

Fabric B

Figure 9. Simulated clothing pressure exerted by fabric $A$ and $B$ in dynamic cycling condition with optimized garment patterns 
clear in Figure 7, the static clothing pressures are lower after pattern customization for both fabrics. Based on the different fabric properties, the dynamic clothing pressure measured and simulated with the customized patterns shows, in general, lower values than in the case of the original pattern (Figure 10). This shows that the comfort of wearing the entire garment is better after the optimization of several patterns.

With respect to the cycling postures, from the graph shown in Figure 11, at the lumber region, armpits and side panel pressure are equal for fabrics $A$ and $B$, while at the collar, sleeve, shoulder, lower front, and neckline region we found higher differences in the pressure of fabric $B$ than $A$, which drops a lot. The body part circumferences decreased. However, this is not the case for all regions. At the armpits, the pelvic region, and the lower chest region fabric $A$ also shows an equally large drop in pressure. Therefore, this difference is due to dimensional deformation of compression fabric and shape of the bodies [37, 38]. Alongside bending (Weft-Warp), adjusting what percentage of total bending intensity to use to decide the buckling stiffness of the corners of fabric. In other words, the higher the buckling ratio, the harder it is to fold the corners of fabric; on the other hand, the lower the ratio, the easier it folds. From the result, it's clear that cotton fabric B can drop or increase in pressure very fast and hence is less suited for sports. Yet, changing the material type does not change the drape of the fabric. For instance, as shown in Figure 11, the dynamic pressures at collar increase significantly compared with the pressures in the static condition, which indicates that the wearing comfort of this part deteriorates slightly, particularly around the front neckline and top edge of the collar line.

A two-sample $t$-test performed to determine if there was a significant difference between the static position and cycling posture (Table 3). In case of Fabric A, with the mean pressure value, there was no significant difference between static position and cycling posture at location of Collar, Neckline, Lower front, Sleeve, Lower chest, Side panels, Pelvic and Lumber region $(p>0.05)$. For our results, to find $P(T \leq t)$, a two-tail test was used, which gives the $p$-value for the twotailed form of the $t$-test. From the data, it is clear that the $p$-value is greater than the standard significance level of 0.05 . On the

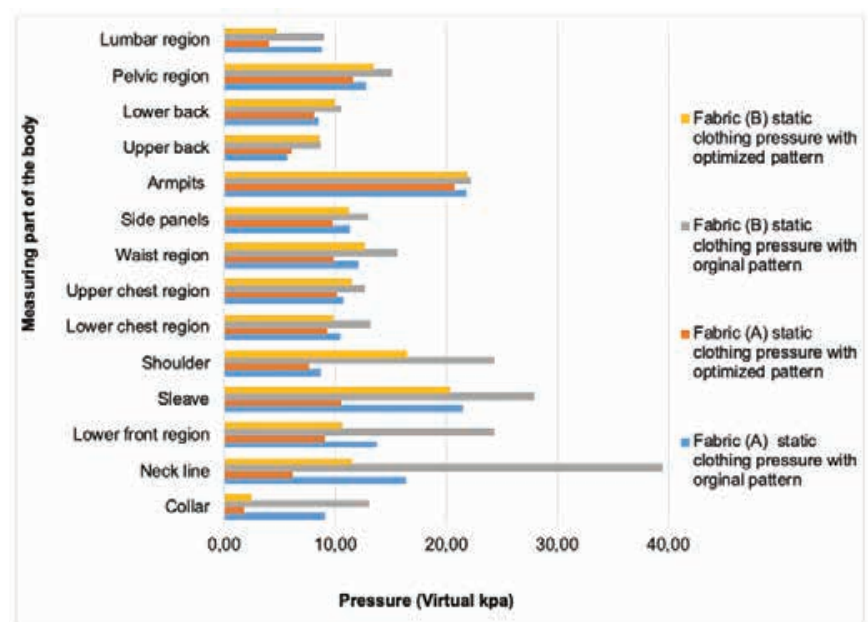

Figure 10. Comparison of pressure in static posture by pattern type for fabric $A$ and $B$ contrary, at shoulder, upper chest, waist region, armpits, upper back and lower back there was a significant difference between the static position and cycling posture, which, from the $p$-value, is less than the standard significance level of 0.05 . These data support the hypothesis that the population means are different. For fabric $B$, there was no significant difference between the static position and the cycling posture except in the shoulder, upper chest, and waist region. In other words, the number of locations where significant pressure differences caused by posture were noticed was lower in case of cotton fabric $B$ as compared with fabric $A$. In spite of that, the pressure exerted by fabric $B$ was higher than by fabric $A$, in both a static and a dynamic posture, and this might be caused by its different properties among which higher density, stretch, and friction.

Generally, the dynamic pressure at all the points of the back increased compared with static pressure. This is because the forward incline of the upper body and the humped back led to an increase in the pressure during cycling. The variation of dynamic clothing pressure on the back is unnoticeable (figure 9). This indicated that the dynamic pressure at the whole back was relatively stable as cycling continued, since the body moves little except for the legs during cycling. Moreover, most body areas decreased and resulted in decreasing pressures. However, the collar shows a large increase in pressure, as do most of the lower body parts: waist, lower back, and the lumbar, slightly. In practice, the cyclist will lean forward during the cycling. The posture and movement of the cyclist will give support to cycling wear to glide forward. Moreover, buttons or zippers, as well as topstitch properties, can change the drape of the fabric as well as the pressure.

In general, the evaluation above demonstrates that customization of the pattern can lead to significant improvement of clothing pressure in static and dynamic condition and can potentially improve the comfort in wearing a cycling garment. Besides, fabric properties and body locations were the main reasons for the variation in pressure distribution. These results give an insight into the parameters that affect the degree of compression applied by the garment for the desired application. A physical prototype will confirm these findings.

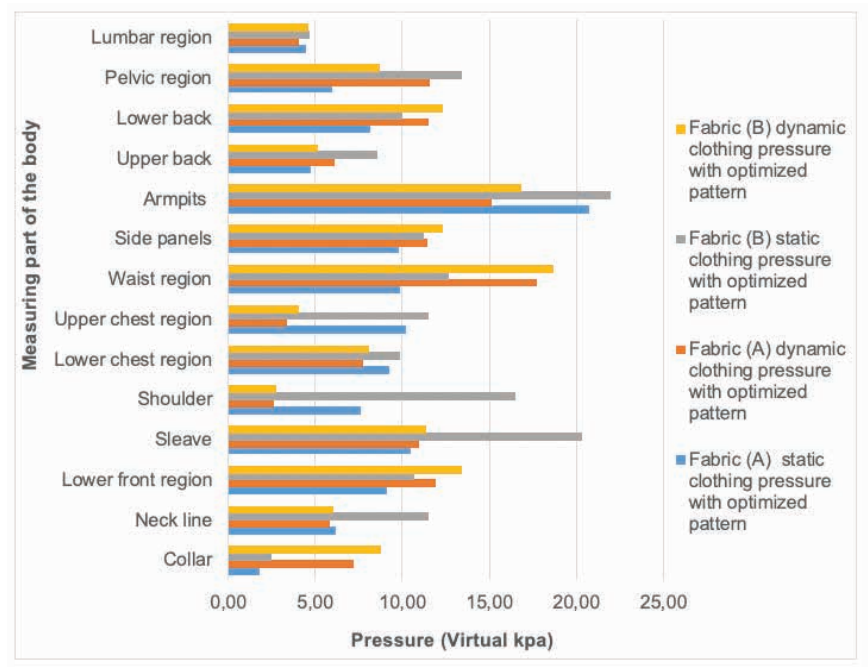

Figure 11. Clothing pressure in posture based on fabric properties of garment with optimized patterns. 
Table 3. Comparison pressure value between static condition and cycling position

\begin{tabular}{|c|c|c|c|c|c|c|c|c|c|c|}
\hline \multirow[b]{2}{*}{$\begin{array}{l}\text { Body } \\
\text { Location }\end{array}$} & \multicolumn{2}{|c|}{$\begin{array}{l}\text { Mean value }(\mathrm{kPa}) \\
\text { for Fabric } A\end{array}$} & \multicolumn{3}{|c|}{$\begin{array}{c}\text { t-Test: Paired Two Sample for } \\
\text { Means }\end{array}$} & \multicolumn{2}{|c|}{$\begin{array}{l}\text { Mean value }(\mathrm{kPa}) \\
\text { for Fabric } \mathrm{B}\end{array}$} & \multicolumn{3}{|c|}{$\begin{array}{c}\text { t-Test: Paired Two Sample for } \\
\text { Means }\end{array}$} \\
\hline & $\begin{array}{l}\text { Static } \\
\text { state }\end{array}$ & $\begin{array}{l}\text { Cycling } \\
\text { posture }\end{array}$ & t Stat & t Critical & P-value & $\begin{array}{l}\text { Static } \\
\text { state }\end{array}$ & $\begin{array}{l}\text { Cycling } \\
\text { posture }\end{array}$ & t Stat & t Critical & P-value \\
\hline Collar & 1.82 & 7.21 & 1.801 & 2.776 & 0.146 & 2.50 & 8.76 & 0.576 & 2.776 & 0.596 \\
\hline Neck line & 6.16 & 5.85 & 2.776 & 2.776 & 0.835 & 11.49 & 6.01 & 2.194 & 2.776 & 0.093 \\
\hline Lower front & 9.12 & 11.94 & 2.371 & 2.776 & 0.077 & 10.67 & 13.40 & 1.375 & 2.776 & 0.241 \\
\hline Sleeve & 10.50 & 10.99 & 0.354 & 2.776 & 0.741 & 20.35 & 11.36 & 2.209 & 2.776 & 0.092 \\
\hline Shoulder & 7.60 & 2.65 & 2.819 & 2.776 & 0.048 & 16.50 & 2.74 & 2.827 & 2.776 & 0.047 \\
\hline Lower chest & 9.28 & 7.75 & 1.810 & 2.776 & 0.145 & 9.84 & 8.09 & 1.377 & 2.776 & 0.241 \\
\hline Upper chest & 10.20 & 3.38 & 4.111 & 2.776 & 0.015 & 11.52 & 4.07 & 5.135 & 2.776 & 0.007 \\
\hline Waist & 9.87 & 17.75 & 3.650 & 2.776 & 0.022 & 12.66 & 18.68 & 4.008 & 2.776 & 0.016 \\
\hline Side panels & 9.77 & 11.44 & 0.513 & 2.776 & 0.635 & 11.22 & 12.34 & 0.338 & 2.776 & 0.752 \\
\hline Armpits & 20.76 & 15.10 & 2.987 & 2.776 & 0.040 & 21.93 & 16.83 & 1.545 & 2.776 & 0.197 \\
\hline Upper back & 4.75 & 6.09 & 13.551 & 2.776 & 0.000 & 8.58 & 5.14 & 2.046 & 2.776 & 0.110 \\
\hline Lower back & 8.14 & 11.51 & 3.919 & 2.776 & 0.017 & 9.99 & 12.29 & 2.717 & 2.776 & 0.053 \\
\hline Pelvic & 5.99 & 11.58 & 2.503 & 2.776 & 0.067 & 13.42 & 8.70 & 2.279 & 2.776 & 0.085 \\
\hline Lumbar & 4.43 & 4.06 & 0.458 & 2.776 & 0.671 & 4.67 & 4.59 & 0.087 & 2.776 & 0.935 \\
\hline
\end{tabular}

\section{CONCLUSION}

This research study analyzed the pressure and fit of a cycling T-shirt in both static (A-pose) and dynamic state (cycling position). The results of the work mainly provide a relevant and useful reference to predict garment fit and evaluation using digital clothing pressure as determined by the CLO 3D software. This software is used particularly to analyze garment pattern design and the impact of fabric mechanical properties from the aspect of clothing pressure for enhancing functional wear comfort, especially to keep the spin in a good position. The result of digital clothing pressure values measured in different body zones exhibits appropriate improvements in relation to the product regarding the evaluation of effectiveness and flexibility of execution of small adjustments for the intended application. With these tools, making a prior evaluation of the materials draping on the body is also possible, since the virtual representation shows the formation of surface texture, volume, and deformation, according to the cut of the pattern and the physical characteristics of the simulated fabric. However, for more realistic simulations of virtual garment fit and effective simulation of the fabric draping, it is necessary to measure the actual fabric's physical textile properties, and this should be used to guide the input to the program. Unfortunately, the difficulty of the simulation software and limited possibilities to use customized fabrics outside of the library makes existing systems difficult to implement and limits the quality of the simulation. The results show the potential of this approach and of the software capability to support the initial development phase of tight-fitting clothing, though the number of elastic fabrics available in the library is very limited. Especially for sportswear, which should fit not only in A or T-pose or on a mannequin, but also in dynamic sports positions, evaluation of fit through software can revolutionize the branch. Nevertheless, at this moment, this technique does not yet eliminate the need to produce the physical prototype garment as a final evaluation of the development process; instead, it largely increases the progress during the initial development.

\section{ACKNOWLEDGMENTS}

The authors would like to give special thanks to Shints ETP Garment Plc, Own Korean Based Factory, for allowing the use of LECTRA software for developing the 2D pattern at their company. The authors also would like to thank Yordanos B., Yoseph D. and Fuad from the Faculty of Apparel Technology, Department of Fashion Design, Bahir Dar University, for their assistance in the simulation software. 


\section{References}

[1] Gascon, S. S., Gilmer, G. G., Hanks, M. M., Washington, J. K., and Oliver, G. D. (2018). Biomechanical influences of a postural compression garment on scapular positioning. Int. J. Sports Phys. Ther., 13(4), 700-706, doi: 10.26603/ ijspt20180700.

[2] Venkatraman, P. D. (2015). Compression garments in sportswear: Case studies to explore the effect of body type, tactile sensation and seam position in garments. IndoCzech International Conference on the Advancements in Speciality Textiles and Their Applications in Material Engineering and Medical Sciences, (ICIC), KCT, India, 2930th April 2014, 1-4, doi: 10.13140/RG.2.1.1531.6001.

[3] Venkatraman, P. D., Tyler, D. J., Ferguson-Lee, L., and Bourke. (2013). Performance of compression garments for cyclists. International Conference on Advances in Functional Textiles 25-26th July 2013 Chancellor's Hotel and Conference Centre, Manchester, UK, 1-32.

[4] Gokarneshan, N. (2017).Design of compression / pressure garments for diversified medical applications. J. Sci. Tech. Res., 1(3), 806-813, doi: 10.26717/ BJSTR.2017.01.000309.

[5] Engel, F., and Sperlich, B.. (2016). Compression garments in sports: Athletic performance and recovery. Switzerland: Springer International Publishing.

[6] Ramelet, A. (2002). Compression therapy. Am. Soc. Dermatologic Surg., 28(1) 6-10.

[7] Partsch, H. (2012). Compression therapy: Clinical and experimental evidence. Ann. Vasc. Dis., 5(4) 416-422, , doi: 10.3400/avd.ra.12.00068.

[8] Partsch, H., Compression therapy. (2013). Pract. Approach to Manag. Treat. Venous Disord., 9,781.447,128, 161-172, doi: 10.1007/978-1-4471-2891-5_17.

[9] Moffatt, C. (2008). Variability of pressure provided by sustained compression. Int. Wound J., 5(2), 259-265, doi: 10.1111/j.1742-481X.2008.00470.x.

[10] Liu, R., Kwok, Y. L., Li, Y., Lao, T. T. H., Zhang, X., and Dai, X. Q. (2005). Objective evaluation of skin pressure distribution of graduated elastic compression stockings. Dermatologic Surg., 31(6), 615-624, doi: 10.1097/00042728-200506000-00001.

[11] Chattopadhyay, R., and Bera, M. (2017). Prediction of pressure due to elastic fabric tube following energy principle. J. Text. Eng. Fash. Technol., 2(5), doi: 10.15406/ jteft.2017.02.00075.

[12] Zhang, X., Yeung, W., and Li, Y. Numerical simulation of 3D dynamic garment pressure. Text. Res. J., 72(3), 245252.

[13] Yeung, K. W., Li, Y., and Zhang, X. (2004). A 3D biomechanical human model for numerical simulation of garment-body dynamic mechanical interactions during wear. J. Text. Inst. Part 1 Fibre Sci. Text. Technol., 95(1-6), 59-79, doi: 10.1533/joti.2001.0050.

[14] Chi, Y., Tseng, K., Li, R., and Pan, T. (2018). Comparison of piezoresistive sensor to PicoPress ${ }^{\circledR}$ in in-vitro interface pressure measurement. Phlebology, 33(5) 315-320, doi: $10.1177 / 0268355517705292$.

[15] Wiegand, C., et al. (2018). Optimized protocol for the biocompatibility testing of compression stockings and similar products with close skin contact in vitro. J. Text. Inst., 5,000, 1-12, doi: 10.1080/00405000.2017.1383640.
[16] Electronica Microlab. PicoPress Technical Manual Rev.6. (2019). Italy, 1-11.

[17] Brophy-Williams, N., Driller, M. W., Halson, S. L., Fell, J. W. and Shing, C. M. (2014). Evaluating the Kikuhime pressure monitor for use with sports compression clothing. Sport. Eng., vol. 17, no. 1, pp. 55-60, doi: 10.1007/s12283-0130125-z.

[18] Tanja, P., and Rudolf, A. (2012). 3D virtual prototyping of clothing products. Innov. Cloth. Technol. Meas. Tech., (September) 28-41.

[19] Bruniaux, P., Kamalha, E., and Torino, P. (2017). Fit evaluation of virtual garment try-on by learning from digital pressure data. Knowledge-Based Syst., 133(July), 174182, doi: 10.1016/j.knosys.2017.07.007.

[20] Jäger, M.-H. (2018). Evaluation and validation of clo $3 d$ fashion design software evaluation and validation of CLO 3D. Tallinna.

[21] Mattila, H. Digital fashion - how and when? (2016). 8th International Textile, Clothing \& Design ConferenceMagic World of Textiles, 1-6.

[22] Gill, S. (2016). Body scanning and its influence on garment development. In: Venkatraman, S. G. H. \& P. (Ed.). Materials \& technology for sportswear and performance apparel, Taylor \& Francis Group (New York).

[23] Sayem, A. S. M., Kennon, R., and Clarke, N. (2010). 3D CAD systems for the clothing industry. Int. J. Fash. Des. Technol. Educ., 3(2), 45-53, doi: 10.1080/17543261003689888.

[24] Li, Y., and Dai, X. Z., and Dai, X.-Q. (2006). Textile biomechanical engineering. In: Y. L. and X. Q. Dai,. (Eds.). Biomechanical engineering of textiles and clothing (1 ed.). Woodhead Publishing Limited \& CRC Press LLC (Cambridge, England).

[25] Kuzmichev, V. E. (2019). Sizing and fit for pressure garments. In: Zakaria, N. and Gupta, D. (Eds.) Anthropometry, Apparel Sizing and Design (2ed.) Elsevier (Duxford, United Kingdom).

[26] Vasile, S., Cools, J., De Raeve, A., Malengier, B., and Deruyck, F. (2019). Effect of rowing posture on body measurements and skin-sportswear interface pressure and implications on garment fit. J. Ind. Text., 1-19, doi: 10.1177/1528083719877005.

[27] Liu, R., Kwok, Y. L., Li, Y., Lao, T. T., and Zhang, X. (2007). Skin pressure profiles and variations with body postural changes beneath medical elastic compression stockings. Int. J. Dermatol., 46(5), 514-523, doi: 10.1111/j.13654632.2007.03175.x.

[28] Wong, X. S. W., Li, A., and Zhang, Y. (2004). Influence of fabric mechanical property on clothing dynamic pressure distribution and pressure comfort on tight-fit sportswear. J. Fiber Sci. Technol., 60(10) 293-299, doi: 10.5923/j. materials. 20160606.01.

[29] Kuzmichev, V. E., Tislenko, I. V., and Adolphe, D. C. (2019). Virtual design of knitted compression garments based on bodyscanning technology and the three-dimensional-totwo-dimensional approach. Text. Res. J., 89(12) 2,4562,475, doi: 10.1177/0040517518792722.

[30] CLO 3D/Marvelous Designer Manual.

[31] Marvelous Designer 2 and CLO 3D 2011 Manual, 2011, pp. 1-27. 
[32] Decaudin, P., Julius, D., Wither, J., Boissieux, L., and Sheffer, A. Virtual garments: A fully geometric approach for clothing design. 25(3), 625-634, doi: https://doi. org/10.1111/j.1467-8659.2006.00982.x.

[33] Jariyapunya, N., and Musilová, B. (2019). Predictive modelling of compression garments forelastic fabric and the effects of pressure sensor thickness. J. Text. Inst., 110(8), 1,132-1,140, doi: 10.1080/00405000.2018.1540285.

[34] Gatti, O., Martiniani, G., and Gaffurini, P. (2017). Introduction to cycling biomechanics and bikefitting. pp. 2-114. Web site: https://www. slideshare.net/OmarGatti/ introduction-to-cycling-biomechanics.

[35] Retul bike, (2011). Retul bike at by studeo DNA - Part 1 crank cycling Retrieved August 10, 2020. Web site: https:// www.crankcycling.com/blog/studeodna.
[36] Retul fit, (2020). Retul or go(niometer) it alone? Slowtwitch. com, DAN EMPFIELD, . https://www.slowtwitch.com/Bike Fit/Bike Fit Equipment and Tools/Retul or go niometer it alone 1174.html (accessed Aug. 10, 2020).

[37] Liu, K. E., et al. (2016). Optimization design of cycling clothes' patterns based on digital clothing pressures. Fibers Polym., 17(9) 1,522-1529, doi: 10.1007/s12221016-6402-2.

[38] Ecole, K. L. I. U., and Ecole, H. Y. (2016). Development of upper cycling clothes using $3 D$-to-2D flattening technology and evaluation of dynamic wear comfort from the aspect of clothing pressure. Int. J. Cloth. Sci. Technol., 28 (6) 736749, doi: 10.1108/IJCST-02-2016-0016. 